\title{
Challenges of lung transplantation in India
}

\author{
Unmil B. Shah ${ }^{1} \cdot{\text { Pradeep } \operatorname{Kumar}^{1} \text { (I) } \cdot \text { Vijil Rahulan }^{1} \cdot \text { Prabhat Dutta }^{1} \cdot \text { Sandeep Attawar }}^{1}$ \\ Received: 25 November 2020 / Revised: 14 February 2021 / Accepted: 17 February 2021 / Published online: 24 April 2021 \\ (C) Indian Association of Cardiovascular-Thoracic Surgeons 2021
}

\begin{abstract}
Lung transplantation (LTx) is the definitive treatment for select progressive end-stage lung disease patients despite being on optimum medical therapy. Asian countries like China, Japan and India have started LTx programmes despite various odds and challenges. Some of these challenges seem to be unique to developing countries. We have elaborated the challenges of LTx in India and their proposed solutions and shared our experience in setting up a successful LTx programme.
\end{abstract}

Keywords Lung transplant $\cdot$ Challenges $\cdot$ India $\cdot$ Solutions $\cdot$ Donor management $\cdot$ Organ donation network

\section{Introduction}

Lung transplantation (LTx) in humans was first attempted by James Hardy in 1963. In 1983, Joel Cooper (Toronto Group) reported the first successful single lung transplant in humans [1].

Since then, LTx has come a long way worldwide overcoming medical challenges of rejections, infections, anastomotic wound healing and immune suppression as well as nonmedical challenges like organ donation awareness and cost.

The first successful heart-lung transplant in India was performed in Chennai [2]. The initial experience was encouraging, paving a way for the new era of thoracic organ transplantation in India. Since then, few centres in India have tried to establish lung transplant programme but it came with many challenges. We have started our thoracic organ transplant programme in February 2017 in Chennai and subsequently we

Pradeep Kumar

dr.pradeep.kumar.singh@gmail.com

Unmil B. Shah

drunmilshah@gmail.com

Vijil Rahulan

Vkrahulan@gmail.com

Prabhat Dutta

prabhatparna@yahoo.co.in

Sandeep Attawar

attawar.sandeep@gmail.com

1 Institute of Heart and Lung Transplant, Krishna Institute of Medical Sciences, Hyderabad, Telangana, India expanded our programme to Bangalore, Mumbai and Hyderabad. Since inception of our programme, we have done 140 lung transplants, 77 heart transplants, and 25 left ventricular assist device (LVAD) implantations until October 2020. We are going to highlight the challenges faced by our unit to establish a successful and viable transplant programme in India. The problems faced by the lung transplant units in India include donor availability, late referrals, reluctance of physicians to refer cases due to lack of confidence in surgical outcomes, ventilator-associated pneumonia, graft dehiscence, infections (bacterial, fungal, viral, mycobacterial), both in the recipient and the donor, logistic issues, cost and organizational issues. Selection of the lung for lung transplant and the correct recipient is crucial for a successful lung transplant. A consensus document for selection of lung transplant candidates has been detailed by the International Society for Heart and Lung Transplant (ISHLT) 2015 and similarly, a consensus statement for donor lung procurement has been released by ISHLT (2020).

\section{Current situation}

Lung transplant has become a definitive treatment option for select end-stage lung disease patients. A national apathy associated with these diseases also exists, as the treatment options are resource-consuming, limited and expensive. Understandably, the remedy by eventual organ replacement remains only a dream for the vast majority of these patients. The reasons for our cadaveric programme not taking off are many and complex. The most obvious ones are social and religious beliefs and taboos, as well as the absence of a decision-making process in the bereaved 
family. Multi-cultural, racial, religious beliefs and diverse traditions in many centuries have resulted in reluctance in organ donation. However, recently, in some parts of the country, organ donation is on the rise.

Despite these challenges, there have been successes seen in cadaver donation in India, which have been due to the efforts of certain dedicated organizations like TRANSTAN (Transplant Authority of Tamil Nadu) in the state of Tamil Nadu, ZTCC (Zonal Transplant Coordination Centre), Mumbai \& Pune in the state of Maharashtra and Jeevandaan in the state of Telangana. India has now a well-structured organ procurement organization (OPO) present at the national level, i.e. National Organ and Tissue Transplant Organization (NOTTO); regional level, i.e. Regional Organ and Tissue Transplant Organization (ROTTO); state level, i.e. State Organ and Tissue Transplant Organization (SOTTO); and city level, e.g. ZTCCs. However, there are issues to be resolved, and therefore organ donation and transplant are still at an early stage in India. The challenge for the success of an organ transplant programme is to build up greater trust in the processes of organ donation and allocation, considering that each donor can save up to 8 lives.

In 2019, the organ donation rate in India was a lowly 0.52 per million population, compared with 36.07 donors per million in the United States of America (USA) [3]. Sensitization of doctors regarding brain stem death declaration is one of the biggest challenges encountered by the transplant programme in India.

The various factors that have made a difference leading to improvement in organ donation are the proactive role of the government in implementation of the organ retrieval act, the role of the media in highlighting the generosity and courage of the donor families and the stories of those who had successful operations, the role of the police in cutting down red tape and also facilitating speedy transfers of organs through crowded roads ('the green corridors'). Physician-patient partnerships have made progress in their goal to 'popularize the cause of organ donation' by distributing donor cards and educational materials about organ donation, and by providing patients with transplant programme information and support. For example, there have been success stories of organ donation in the state of Maharashtra, giving a new lease of life to 449 patients in 2019, with it receiving the Best State Award by NOTTO on the 10th Indian Organ Donation Day [4].

A major change in the transplant laws can significantly increase the deceased donor pool if declaration of brain stem death is made mandatory for every hospital.

The limitations associated with organ transplantation start from donor availability, to recipient optimization and preparation, to logistical and expertise challenges. An 'ideal' system is an aim, but the geographical and logistical aspects must be taken into consideration. In India, LTx is evolving and there have been challenges which are unique to the developing countries. The aim of the review is to discuss the challenges and their possible solutions.

\section{Challenges}

\section{At donor level}

On the availability of possible donors, the common basic question facing any lung transplant unit is 'can we use these lungs?' The characteristics of the 'acceptable' organ donor have been established and are listed in a consensus statement [5] as described by Copeland et al. (Table 1).

These are the so-called ideal donor criteria. But all donor calls do not fit into these criteria. So, there was a need to create the so-called extended donor criteria. As per a review by Meers et al., out of 164 effective multiple organ donors (MODs), 56 (34.1\%) were accepted as lung donors, whereas $108(65.9 \%)$ were rejected. The primary reasons for declining were abnormal chest $\mathrm{X}$-ray findings $(20 \%)$, older age $>70$ years $(13 \%)$, poor oxygenation $\left(\mathrm{PO}_{2} / \mathrm{FiO}_{2}<300 \mathrm{mmHg}\right)$ $(10 \%)$ and airway aspiration (9\%). Most of these rejected donors presented with a combination of risk factors. Donors were divided into standard (25) and extended (31) donors according to the criteria. The majority of extended donors had a smoking history $>20$ pack years $(12 / 31)$ or abnormalities on chest X-ray (10/31). On the other hand, oxygenation index was below $300 \mathrm{mmHg}$ in only $2 / 31$ accepted lungs. Out of the $56(34.1 \%)$ accepted lung donors, 50 transplants were performed at the centre, 23 from standard criteria donors versus 27 from extended criteria donors. There were no significant differences in hospital outcome and in early survival between lung recipients from both donor groups [6].

Thus, one of the challenges remains about managing 'ideal' donors. If the donor lung falls into extended criteria, the next challenge is to optimize the donor lungs. For a successful outcome in a lung transplant unit, it is important to have an aggressive donor lung management strategy.
Table 1 Characteristics of an ideal donor 
The majority of lung transplant programmes are conservative in selecting donor lungs, resulting in a wait-list mortality rates as high as $12.8 \%$ per year (USA) and $21 \%$ per year (United Kingdom-UK) [7]. The lack of organ donors is a significant problem for patients awaiting LTx, because lung retrieval rate is only 10 to $20 \%$ of organ donors [8]. As per Mohan Foundation [9], out of 875 deceased donors in India in 2018, 191 lungs were retrieved. The key to increasing the lung donor pool lies in (a) optimizing MOD management with judicious care for potential organs to be donated, which should be approached as a strategy to avoid losing donors to inadequate protocols, (b) utilizing lungs after donation after circulatory death (DCD), (c) using ex vivo lung perfusion (EVLP) and (d) if possible, using lobar lung living donors [7]. A solution to the imbalance between the number of lung transplant candidates and the limited availability of organs is to consider marginal donors. Optimization by EVLP in explanted lungs and TransMedics organ care system (OCS) for heart and lung transportation is a new and promising approach that some heart and lung transplant centres have initiated.

In view of more number of extended criteria donors, there was a need for research regarding expanding donor pool. Hence, came 2 game-changing concepts which when applied were able to increase donor pool, helping in turn to reduce wait-list mortality. These are (a) EVLP and (b) DCD.

EVLP This is a technique used to evaluate and screen compromised donor lungs with potential for recovery. This technique has been already used in LTx centres across the world. EVLP can restore the circulation and ventilation of the ex vivo lung. At an ambient temperature of $37^{\circ} \mathrm{C}$, a membrane oxygenator is used to simulate oxygen consumption in the body via deoxygenation and maintain the physiological state of lungs with specific perfusate and ventilation. The Steen solution is currently the only Food and Drug Administration (FDA)-approved EVLP perfusate for clinical use. The ventilation gas in the lung membrane consists of $\mathrm{N}_{2}(86 \%), \mathrm{CO}_{2}(8 \%)$ and $\mathrm{O}_{2}$ $(6 \%)$. The hypoxic gas mixture removes the oxygen in the circuit to simulate oxygen consumption in the body. The EVLP system includes a ventilator, an endotracheal tube, the perfusate and a fluid circuit, a reservoir, an oxygenator, a pump and a thermostat as elaborated by Pan et al. [10]. EVLP is currently used mainly to evaluate certain high-risk donor lungs. It is mainly indicated for (I) an oxygenation index $<300 \mathrm{mmHg}$; (II) pulmonary oedema as indicated by the last chest X-ray; (III) collapse or poor expansion of a donor lung during harvest; (IV) blood transfusion $>10 \mathrm{U}$; and (V) lungs from donors with cardiac death. EVLP is not suitable in cases of apparent pneumonia, severe mechanical lung injury (including multiple lobar injury) or significant aspiration of gastric contents.
Zhang et al. [11] have shown that transplantation of the previously discarded lungs, recovered by EVLP, leads to equal outcomes compared to conventional LTx methods. The 3-year survival was 78\% (7/9) (EVLP group) versus $83 \%$ (15/18) (non-EVLP group).

DCD Here, organs are removed from donors after cardiac arrest. DCD can be considered in a patient who does not fulfil brainstem death criteria, but has no hope of recovery, and it is in the best interest of the patient to withdraw life-sustaining treatment. The main difference between DCD and donation after brainstem death (DBD) organs is the warm ischemia time. Warm ischemia will be minimal in DBD donors as compared to DCD donors, where there is an interval after asystole where organs are not being perfused and have not yet been cooled, leading to relatively longer warm ischemia time. In India, the concept of DCD is evolving, with want of more legal clarity regarding withdrawal of life support. When decision to withdraw treatment support has been taken by the family and the physician, discussion regarding organ donation can be initiated. Selective use of EVLP is a part of the DCD programme in most centres. Broadly, DCD donors are divided into controlled and uncontrolled donors (modified Maastricht classification) as mentioned in the review by Inci [12]. Van Raemdonck et al. [13] in their study cohort of 11,516 lung transplant patients with 5-year follow-up have shown similar excellent long-term survival in DCD-III and DBD lung donor recipients in 23 experienced centres. On multivariate analysis, transplant diagnosis (bronchiectasis vs. pulmonary fibrosis; HR: 1.48 [1.21, 1.80]; and other indications vs. pulmonary fibrosis: HR 1.22 [1.09, 1.37]), procedure type ([single lung vs. bilateral and double lung]: HR: $1.21[1.11,1.32])$ and transplant era (2003-2009 vs. 2010-June 2016, HR: 1.18 $[1.10,1.27])$ were all independently associated with survival $(p<0.001)$. More importantly, donor type (DCD-III vs. DBD) was not (HR 1.04 [0.90, 1.19]; $p=0.61$ ).

Both the above concepts, which are into clinical practice, are costly affairs as far as Indian perspective is concerned. Bringing EVLP to Indian shores will force lung transplant units to consider costs related to it.

Proposed solutions Managing donors with a multidisciplinary approach and team work would definitely improve the quality. Embracing novel concepts to expand donor pool and judiciously utilizing extended criteria would be the way ahead (Fig. 1).

\section{At recipient level}

It is imperative to have early referrals to transplant clinics to make patients and their relatives sensitive to this modality of treatment for their end-stage lung diseases, which becomes definitive. Lung transplant units in India face significant 
Fig. 1 Proposed solutions to overcome donor-level problems

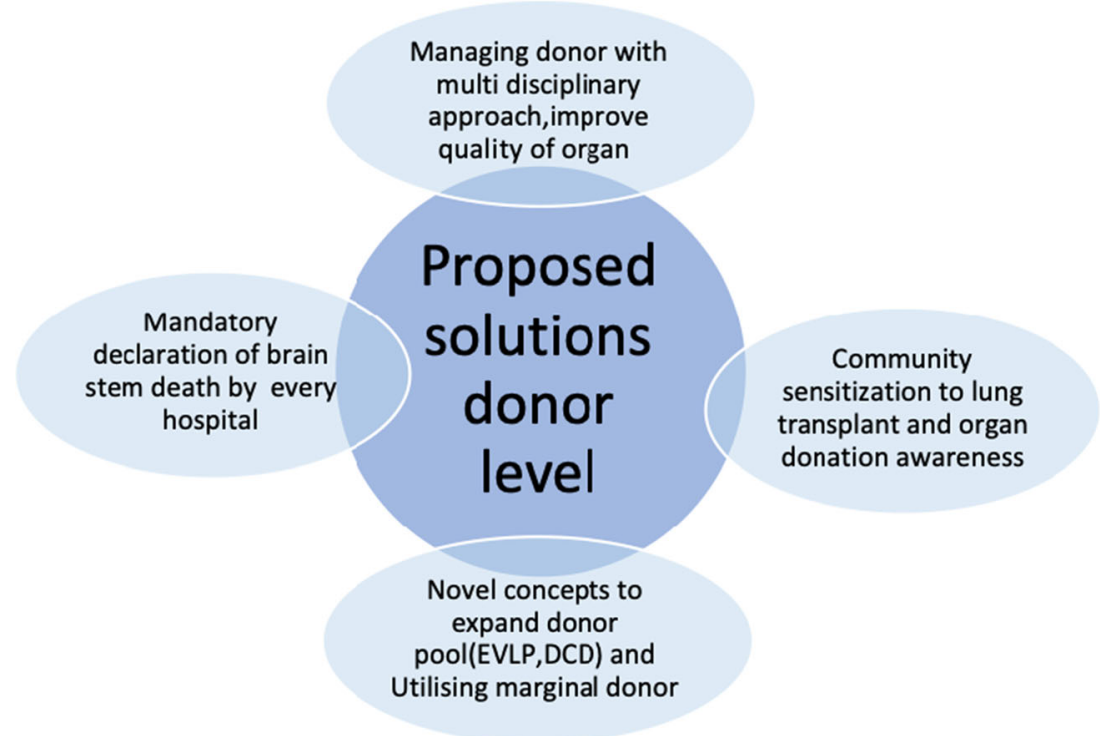

problems with late referral and reluctance of physicians to refer cases due to lack of confidence in surgical outcomes. It is important to bring awareness among medical fraternity about the disease-specific indications required for referral to clinic and listing which are different. Awareness among general public and medical fraternity is required for successful outcomes of lung transplant.

An interesting study by Ramos et al. [14] to analyse predictors of non-referral of patients with cystic fibrosis (CF) for lung transplant evaluation in the USA showed that despite meeting lung function criteria for lung transplant evaluation, $35 \%$ of patients with CF had not yet been referred to any lung transplant centre. Predictors of non-referral included low socioeconomic status, older age and Burkholderia cepacia complex sputum culture [14].

In another study by Martin et al. [15], to evaluate causes of death in French CF patients, the authors demonstrated that of 256 deaths, half occurred after LTx and were related to early or late complications of transplantation, whereas half occurred in patients who did not receive LTx and were primarily related to respiratory failure or massive haemoptysis. Among patients who did not receive LTx, only $19 \%(25 / 129)$ died while waiting on a LTx list. Lack of listing for LTx was primarily related to late or to lack of transplantation referral, rather than to contraindication to transplantation. A striking finding was that $28 \%$ of patients who died without receiving LTx were not proposed LTx, despite the fact that most of these patients had no obvious contraindication to transplantation. These data suggested that improvement in transplantation referral strategies may result in transplantation-related survival benefits [15].

Earlier referral may help give patients enough time for transition to transplant. Early referrals also give time for patients to undergo pulmonary rehabilitation (PR) preoperatively, which impacts the outcome of transplant. In a review by Hoffman et al., it was shown that PR is an effective treatment option for patients on the waiting list for LTx and can improve quality of life and exercise capacity in those patients [16]. In a review by Wickerson et al. [17], authors have proven that exercise training provides an essential role in optimizing functional capacity and fitness pre-transplant, as well as improving outcomes and quality of life post-transplant.

So it becomes imperative to refer early, and that from Indian perspective is challenging, even though scenario is changing with rising awareness about lung transplant. Referring recipients in the so-called transplant window is crucial.

Lack of official uniform scoring system for recipients in India, like the Lung Allocation Score (LAS), which has been used in most successful lung transplant programmes, also poses a practical challenge. The score-which ranges from 0 to 100 , with higher values connoting higher priority for transplantation-has proven to be beneficial. Waiting time and waiting list mortality rates have dramatically decreased since implementation of the LAS in 2005, suggesting that the LAS system has achieved its goal of reducing the number of deaths among lung transplant candidates, as mentioned in the review by Hook et al. [18].

Early referrals also help in correcting nutritional status of patients as most of them have either low body mass index (BMI), or higher BMI beyond acceptable range, which can impact outcome post-transplant. As per ISHLT 2014 consensus statement, class I obesity (BMI $30.0-34.9 \mathrm{~kg} / \mathrm{m}^{2}$ ), particularly truncal (central) obesity, and progressive or severe malnutrition are relative contraindications, while class II or III obesity (BMI $\geq 35.0 \mathrm{~kg} / \mathrm{m}^{2}$ ) is an absolute contraindication for LTx [19]. 
Proposed solutions Early referrals, sensitization to LTx and robust pulmonary rehabilitation programmes would help in better preparation and selection of candidates for LTx. Partnerships with community referring physicians can greatly enhance the continuity of care through all phases of transplantation. There is the need of the day to have a validated official scoring system like the LAS for recipients in India (Fig. 2).

\section{At infrastructure level}

For setting up a lung transplant programme, it is mandatory to have a separate cardiothoracic transplant department/floor to reduce risk of infection. Now with the coronavirus disease (COVID) pandemic worldwide, it has become imperative to have a COVID operating room separate from a non-COVID operating room. Negative pressure operating rooms are becoming the need during this COVID era [20]. Access to extracorporeal membrane oxygenation (ECMO) facilities is a must and an important part of any lung transplant programme.

Another challenge for LTx in India is to have virtual human leukocyte antigen (HLA) crossmatch of donor and recipient like in the West. Lack of capability to conduct a HLA test onsite for donors at remote locations and longer turnaround times for tests are the practical challenges which need to be addressed. Also, to have Indian calculated panel reactive antibodies (cPRA), it will require studies to be conducted in Indian population for standardization. Accepting heart and lung after virtual crossmatch can improve our short- and long-term outcomes further.

Proposed solutions To have a successful LTx programme, infrastructure and expertise availability is the core issue. We should adopt and implement systems to meet global practice. Need is to setup quality controlled HLA labs in each district at least and robust data to develop Indian population cPRA to fill this void in the field of transplantation in India.

\section{At society/community level}

It is important to raise awareness of the society about organ donation. This will help more pledges for donation and in turn expand the donor pool.

Proposed solutions Community awareness programmes should be developed to encourage organ donations. Professional training for family councillors can help create awareness effectively and improve consent rate. Professionally trained transplant coordinators in Iran, for example, report a consent success rate between 83 and $88 \%$ as mentioned in the review by Nguyen et al. [21].

\section{At logistics level}

At times, some donor organs require to be ferried by chartered flights to the transplanting centre in view of geographical and logistical limitations. The cost of chartered flights becomes a challenge and adds on to the total cost, even though nowadays, situation is changing and more people can afford it.

\section{Post-transplant challenges}

A review was done by Nguyen et al. [21] studying various challenges to lung transplant in the developing countries. Various study references were documented, to mention a few:

(a) According to a ISHLT report, primary graft dysfunction (PGD) represents the most common cause of death in the first 30 days after lung transplants, with an overall incidence of $30 \%$. Although infection was the second leading cause of death in the ISHLT report, it may play a larger role in developing countries. Pneumonia is the most common infection and can lead to sepsis and ICU admission. One of the most significant pathogens is
Fig. 2 Proposed solutions to overcome recipient-level problems

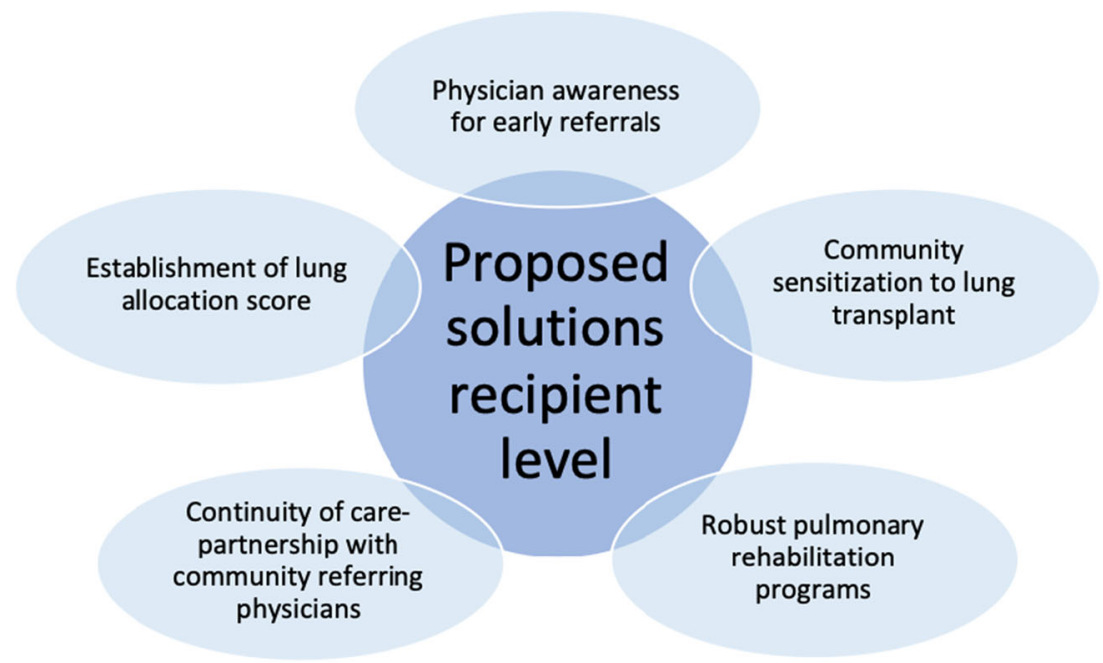


tuberculosis (TB), with prevalence among solid organ transplant (SOT) as high as $15 \%$. TB in SOT has been associated with poor graft and patient survival.

(b) In a study of 2349 transplants in Colombia from 1995 to 2015 , active TB was diagnosed in 31 patients. The development of TB disease post-transplantation is mainly because of the reactivation of latent TB infection (LTBI). The prevalence of LTBI among transplant patients in some developing countries remains high: Korea 33\%, Iran $19 \%$ or Turkey $53 \%$.

Thus, infections pose a unique challenge, some endemic to a particular region, to managing patients postoperatively, in transplant programmes. Managing recipients, staying long distance, with frequent biopsies, therapeutic drug level monitoring, clearance bronchoscopies and airway interventions are some of the challenges recipients might face, but with use of technologies like creating WhatsApp groups to increase more interaction and surveillance can help mitigate some of these challenges. Coordination with local pulmonologists can also help solve some of these issues.

Proposed solutions Managing immunosuppression posttransplant in developing countries may differ from those in developed countries. More data is required to demonstrate whether slightly lower levels of immunosuppression, balancing the risk of rejection, would help post-transplant patients in countries like India, where infections are highly prevalent or endemic.

\section{Team building}

Lung transplant is a team 'sport' as said by Nguyen et al. [21]. Building a team with team players sharing the same passion and interest takes time. No transplant programme can be successfully managed single-handedly. Team building becomes a challenge, especially while starting a new programme. Lack of trained retrieval and transplant surgeons, non-availability of adequate infrastructure and other trained manpower are also major issues. This is why we have few registered transplant centres and very few non-transplant organ retrieval centres.

Involving transplant pathologists/immunologists and wide availability of such expertise pose a challenge with leading transplant centres in India having this expertise. Hence, referring for opinions and coordination is the key, until each and every team acquires such an expertise.

Institutionalizing new approaches, consolidating improvements and introducing more changes can only be possible with team effort.

Proposed solutions Building a multi-disciplinary team consisting of dedicated medical specialists from different disciplines working together, to care for the patient, as well as coordinators and administrators to appropriate and advocate for necessary resources (financial, equipment, space, personnel) to ensure the success of the programme is important, as elicited by Nguyen et al. Continuous efforts to improve skills and knowledge are essential for the team [21].

\section{Organ donation network}

Robust well-oiled organ donation network is important for smooth functioning of transplant programmes. India has a well-structured government network at national level (NOTTO), regional level (ROTTO), state level (SOTTO) and city level (ZTCCs). This has helped in coordinating organ donation at various levels.

Despite diligent efforts of activists in the transplant community, there remains a need for a central regulatory organization, similar to the non-profit scientific and educational United Network of Organ Sharing (www.unos.org), which administers the organ procurement and transplantation network in the USA. Such an organization would enhance infrastructure to increase organ donation and procurement. Another important aspect of successfully developing organ transplantation in India lies in the education of its masses about the importance of donating organs.

\section{Cost of lung transplant programme}

For anyone who is planning to seek transplant, the most important factor will be considering 'the costs'. The cost of the transplant is a major issue especially in developing countries. Public insurance cover is minimal and private insurance too is small and pocket dependent, and hence government policy is needed to help patients in need, who cannot afford the cost of transplant.

\section{Novel COVID-19 challenge}

Since the COVID pandemic hit worldwide, India also saw its ill effects on the rate of organ donation. Organ donation drastically reduced during the lockdown period, with few OPOs like ZTCC Mumbai \& Pune continuing organ donation, albeit less in number. New guidelines were prepared for COVID screening for donors and recipients by ZTCC Mumbai and NOTTO and implemented. Reduction in available donors, known fact of reduced lung retrieval rates as compared to other solid organs and stretched health care resources led to reduction in LTx in India. In such situations, having an EVLP programme would benefit in optimizing marginal lungs for transplantation. The cost of EVLP circuit and Steen's solution etc. are financial challenges which a LTx programme faces in Indian settings. The need for validated indigenous solutions is the probable key to the above problem. The COVID pandemic also made changes in the surgical side with need for negative 
pressure operating rooms (ORs), wearing personal protective equipment (PPEs) and creating separate non-COVID facilities in institutes for transplant care. The COVID pandemic also saw reduced referrals for LTx as well as few wait-listed patients developing severe acute respiratory syndrome coronavirus 2 (SARS-CoV-2) infection. This also impacted wait-list mortality. In our programme, out of 24 patients on wait-list from March 2020 to October 2020, 7 patients (29\%) developed SARS-CoV infection. Out of these 7 patients on waitlist, 3 patients $(42 \%)$ recovered and underwent bilateral LTX successfully, 3 patients expired $(42 \%)$ and 1 patient is awaiting LTx.

Currently, as the COVID pandemic is stabilizing, organ donations have started increasing in India, which is a hope to the wait-listed patients.

\section{Our Indian experience}

We did 132 LTx from April 2017 to March 2020. The age range of subjects was 16 to 71 years with a mean of $48.60 \pm$ 13.45 , with male to female ratio of $1.24: 1$. Interstitial lung disease (ILD) constituted the most common diagnosis among $87(65.91 \%)$ patients, followed by bronchiectasis in 13 $(9.85 \%)$ and primary pulmonary hypertension $(\mathrm{PPH})$ in 9 $(6.82 \%)$ patients. Chronic hypersensitivity pneumonitis and idiopathic pulmonary fibrosis were the common ILDs. Eight $(6.06 \%)$ patients needed preoperative mechanical ventilation and $14(10.61 \%)$ needed preoperative ECMO. Among the study population, $77.3 \%$ participants had bilateral LTx, $14.39 \%$ had heart and lung transplantation (HLTx) and $5.30 \%$ had single LTx. The mean days on ventilator were $7.13 \pm 8.43$, mean ICU stay was $15.76 \pm 11.66$ days and mean duration of hospital stay was $28.61 \pm 22.35$. The overall mortality was $14.4 \%$. One-year mortality was $26(19.7 \%)$ and survival was $80.3 \%$.

\section{Developing countries and LTX}

As per a review article by Aryal and Nathan [22], 1- and 3year survivals post-lung transplant patients are reported at $70.11 \%$ and $61.16 \%$, respectively (China Lung Transplant Registry). In the ISHLT Registry, for the era of 2010-June 2017, the 1- and 3-year survivals were $84.8 \%$ and $70.3 \%$, respectively. This shows that successful outcomes are catching up fast in developing countries to match with Western world data.

Similarly, a review article by Date [23] reported that between 1998 and 2015, LTx was performed in 464 patients (55 children, 419 adults) at 9 lung transplant centres in Japan. (Cadaveric lung transplant was performed in 283 patients (61\%) and LDLLT (Living-Donor Lobar) was performed in
181 patients (39\%).) The 5-year survival was $72.3 \%$ and $71.6 \%$, respectively.

Thus, Asian countries are on fast-track mode for successful outcomes post-LTx.

\section{Conclusion}

Challenges of LTx in developing countries like India are not unsurmountable. Proper coordination between various stake holders, creating awareness in society about lung transplant and organ donation, building a team of dedicated and passionate individuals, early referrals to transplant clinics and better donor management will usher in an exciting future for lung transplant programmes in India.

Funding None.

\section{Declarations}

Conflict of interest The authors declare no competing interests.

Ethical approval This is a review article; no ethical approval is required.

\section{References}

1. Venuta F, Van Raemdonck D. History of lung transplantation. J Thorac Dis. 2017;9:5458-71.

2. Madhu Sankar N, Kurian VM, Rajan S, Ninan B, Ajit M, Cherian KM. Heart-lung transplantation in India: initial experience. Indian Heart J. 2003;55:185-7.

3. Global Observatory on Donation and Transplantation. (WHO) www.transplant-observatory.org.

4. The Indian Express: written by Anuradha Mascarenhas | Pune | March 2, 2020 3:46:53 am.

5. Copeland H, Awori Hayanga JW, Neyrinck A, et al. Donor heart and lung procurement: a consensus statement. J Heart Lung Transplant. 2020;39:501-17.

6. Meers C, Van Raemdonck D, Verleden GM, et al. The number of lung transplants can be safely doubled using extended criteria donors; a single-center review. Transpl Int. 2010;23:628-35.

7. Popov A-F, Sabashnikov A, Patil NP, et al. Ex vivo lung perfusion state of the art in lung donor pool expansion. Med Sci Monit Basic Res. 2015;21:9-14.

8. Miñambres E, Pérez-Villares JM, Chico-Fernández M, et al. Lung donor treatment protocol in brain dead-donors: a multicenter study. J Heart Lung Transplant. 2015;34:773-80.

9. https://www.mohanfoundation.org/deceased-organ-donation-inindia.asp.

10. Pan X, Yang J, Fu S, Zhao H. Application of ex vivo lung perfusion (EVLP) in lung transplantation. J Thorac Dis. 2018;10:4637-42.

11. Zhang ZL, van Suylen V, van Zanden JE, et al. First experience with ex vivo lung perfusion for initially discarded donor lungs in the Netherlands: a single-centre study. Eur J Cardiothorac Surg. 2019;55:920-6.

12. Inci I. Donors after cardiocirculatory death and lung transplantation. J Thorac Dis. 2017;9:2660-9. 
13. Van Raemdonck D, Keshavjee S, Levvey B, et al. Donation after circulatory death in lung transplantation-five-year follow-up from ISHLT Registry. J Heart Lung Transplant. 2019;38:1235-45. https://doi.org/10.1016/j.healun.2019.09.007.

14. Ramos KJ, Quon BS, Psoter KJ, et al. Predictors of non-referral of patients with cystic fibrosis for lung transplant evaluation in the United States. J Cyst Fibros. 2016;15:196-203.

15. Martin C, Hamard C, Kanaan R, et al. Causes of death in French cystic fibrosis patients: the need for improvement in transplantation referral strategies! J Cyst Fibros. 2016;15:204-12.

16. Hoffman M, Chaves G, Ribeiro-Samora GA, Britto RR, Parreira VF. Effects of pulmonary rehabilitation in lung transplant candidates: a systematic review. BMJ Open. 2017;7:e013445.

17. Wickerson L, Rozenberg D, Janaudis-Ferreira T, et al. Physical rehabilitation for lung transplant candidates and recipients: an evidence-informed clinical approach. World J Transplant. 2016;6: 517-31.

18. Hook JL, Lederer DJ. Selecting lung transplant candidates: where do current guidelines fall short? Expert Rev Respir Med. 2012;6: $51-61$.
19. Weill D, Benden C, Corris PA, et al. A consensus document for the selection of lung transplant candidates: 2014-an update from the Pulmonary Transplantation Council of the International Society for Heart and Lung Transplantation. J Heart Lung Transplant. 2015;34: $1-15$.

20. Guidance from the International Society of Heart and Lung Transplantation regarding the SARS CoV-2 pandemic (2020). www.ishlt.org.

21. Nguyen AT, Brzezinski M, Chen J, Nguyen NV, Dinh LV, Kukreja J. Lung transplant programs in developing countries: challenges, solutions, and outcomes. Curr Opin Organ Transplant. 2020;25: 299-304.

22. Aryal S, Nathan SD. Lung transplantation in China: a firm foundation for a solid future. Ann Transl Med. 2020;8:265.

23. Date H. Current status and problems of lung transplantation in Japan. J Thorac Dis. 2016;8:S631-6.

Publisher's note Springer Nature remains neutral with regard to jurisdictional claims in published maps and institutional affiliations. 